\title{
Therapeutic potential of Hsp27 in neurological diseases
}

\author{
Anila Venugopal ${ }^{1}$, Kasthuri Sundaramoorthy ${ }^{2}$ and Balachandar Vellingiri ${ }^{*}$
}

\begin{abstract}
Background: Heat shock proteins (Hsps) are widely reported in normal cellular dynamics under stress and nonstress conditions, and parallelly, the studies regarding its role in disease condition are also progressing steadily. The function of Hsps in neurodegenerative disorders is puzzling and not fully understood. This review aims to focus on the role of Hsp27 in normal and diseased conditions and emphasize its therapeutic potential.

Hsp27: Hsp27, in particular, has shown to be involved in cell viability and actin cytoskeleton remodeling and also shown to improve many disease conditions. Phosphorylated Hsp27 modulates the p53 pathway by downregulating cellular senescence and also lowers reactive oxygen species to protect TNFa-mediated apoptosis. Hsp27 is also known to interfere with mitochondria-dependent and mitochondria-independent cell apoptotic stimulation.

Conclusion: This article will highlight the various functions of Hsp27 especially as an anti-apoptotic factor and stress response factor and its therapeutic potential in preventing neuronal apoptosis in neurological diseases. This review also includes a comparison of the therapeutic potential of Hsp27 with regard to other small Hsps.
\end{abstract}

Keywords: Heat shock proteins, Hsp27, Neurological diseases, Therapeutic

\section{Background}

Heat shock proteins (Hsps) were discovered by Ferruccio Ritossa in 1960 in Drosophila melanogaster. Hsps are a group of proteins expressed by cells in response to any environmental stress. For example, during stressful conditions such as exposure to toxins or hypoxia, Hsps are upregulated to generate proteins that will be identified as antigens to stabilize the condition. Hsps also function as molecular chaperones that are involved in folding and unfolding of different proteins to prevent the aggregation of unwanted protein and facilitate proper refold of damaged proteins. Hsps constitute of many different proteins separated based on their molecular masses. The main Hsps comprise of Hsp100, Hsp90, Hsp70, Hsp60, Hsp40, and small heat shock proteins (sHsps). Small heat shock proteins (HspB1-HspB10) have a molecular weight of monomeric forms between 12 and $43 \mathrm{kDa}$ and are characterized by the presence of $\alpha$-crystallin domain that is flanked by less conserved $\mathrm{N}$-terminal domain and

\footnotetext{
* Correspondence: geneticbala@buc.edu.in

${ }^{1}$ Human Molecular Cytogenetics and Stem Cell Laboratory, Department of Human Genetics and Molecular Biology, Bharathiar University, Coimbatore, Tamil Nadu 641046, India

Full list of author information is available at the end of the article
}

C-terminal extension. Among the sHSPs, Hsp27 (also known as HspB1) has a significant responsibility in increasing the cell viability and acts in multiple roles as an anti-apoptotic protein and antioxidant as well as being involved in actin cytoskeleton remodeling $[1,2]$.

By targeting Hsp27, apoptosis of $\alpha$-synuclein may be prevented and regulated which makes it useful in treating neurodegenerative disorders like Parkinson's disease. With the use of its stress response, it is possible to eliminate the aggregation of amyloid plaques and thus prevent the progression of Alzheimer's disease. Due to its numerous roles within the cell, Hsp27 is a promising therapeutic candidate for neurological diseases. This review discusses the possible therapeutic strategies of Hsp27 against few neurological diseases and also includes a critical comparison of other Hsps having therapeutic activity.

\section{Functional regulation and inhibitory actions of Hsp27}

Cells activate various signaling pathways when exposed to environmental stress conditions. This stress can cause damage to the cellular mechanism by causing mitochondrial dysfunction, protein misfolding, and finally neuronal 
cell death. During stress conditions, Hsp27 stabilizes the cytoskeleton with actin capping to prevent cellular injury. It scavenges the reactive oxygen species (ROS) by raising the levels of intracellular glutathione and is associated with independent and dependent apoptotic pathways in mitochondria. In unstressed cells, these Hsps are actively involved in folding, assembly, intracellular localization, secretion, regulation, and degradation of proteins [3].

The functions of Hsp27 are influenced by posttranslational modifications via phosphorylation with differential effects in cellular functions. The expression of Hsp27 gene during stress is caused by the binding of HSF1 (heat shock transcription factor-1) to HSE (heat shock element) which incorporates the promoters of Hsp27 [4]. During mitosis, HSF2 (heat shock transcription factor-2) binds to HSE to induce normal stressinducible expression of genes [5]. The main role of HSF1 is to induce the expression of Hsps. This can be seen in studies where HSF1 protein levels are elevated in cancer, whereas in neurodegenerative disorders, the levels are depleted. The elevated expression of HSF1 has shown to enhance the pro-survival function in neurodegenerative disorder studies [4].

The expression levels of Hsp27 vary among different cells as well as species based on their sites of phosphorylation. In humans, Hsp27 gets phosphorylated at specific serine residues such as Ser15 (Serine 15), Ser78 (Serine 78), and Ser82 (Serine 82) which acts as a common substrate for MK2 (Mapkap kinase 2), MK3 (Mapkap kinase 3), and MK5 (Mapkap kinase 5), respectively [6, 7]. Hsp27 occurs in two pathways-p38MAPK (p38 mitogen-activated protein kinases) and PKA (p21-activated protein kinases) pathways. MK2, a major Hsp27 kinase, phosphorylates Hsp27 by an activated p38MAPK pathway in response to cellular stress. Similarly MK5, another Hsp27 kinase, also phosphorylates Hsp27 by the PKA pathway [7, 8].

Upon phosphorylation, Hsp27 has a role in actin filament regulation where it promotes polymerization contributing to microfilament network maintenance by preventing filament degeneration and also blocking the early response of actin to growth factors [9]. In the unphosphorylated form [10], Hsp27 has an alternative role in actin capping thereby inhibiting assembly of wild-type proteins [11]. During platelet activation, Hsp27 undergoes conformational changes by phosphorylation, promotes interaction between Hsp27 and actin or between Hsp27 and other actin-associated proteins, and facilitates translocation of proteins to the cytoskeleton [12].

Hsp27 phosphorylation also modulates the p53 pathway by inhibiting the accumulation of p 21 which downregulates cellular senescence. p21 is an inhibitor of cyclin-dependent kinases that are important for the progression of the cell cycle. On the inhibition of Hsp27 phosphorylation, an increased accrual of p21 may occur, due to decreased p53, leading to apoptosis [1]. During stress conditions, as an antiapoptotic agent, Hsp27 expression inhibits mitochondrial injury and apoptosis of cells [13]. When Hsp27 expression is suppressed, it will lead to an increase in cellular susceptibility to apoptosis contributing to organ dysfunction [14]. This makes Hsp27 hold a pivotal post that can control cell death and cell survival. Hsp27 interferes with the apoptotic signaling pathway by interacting with both mitochondriadependent and mitochondria-independent pathways (Fig. 1). It affects different key components of the apoptotic pathway in various ways as follows: (a) It enhances PI3-K which activates Akt-Bax interaction, inhibiting Bax activation and translocation to mitochondria preventing the release of cytochrome $\mathrm{c}$ from mitochondria [14]. (b) It inhibits the Ask1-JNK pathway leading to cytochrome c leakage [15]. (c) It prevents apoptosome formation [16]. (d) It interacts with Daxx in the nucleus preventing its translocation to cytosol which is essential for interaction with the Fas receptor involving apoptosis [17]. (e) Hsp27 seems to be involved in inhibition of the Smac from mitochondria, and this may lead to inhibition of caspases [14]. (f) It also downregulates tBID released from caspase 8 [16].

Besides its activity in apoptosis inhibition, Hsp27 also acts as an antioxidant by lowering the levels of ROS and iron by raising intracellular glutathione levels [18]. ROS plays a role in intracellular signaling and regulation and acts as redox messengers at cellular concentrations. Under normal conditions, ROS is produced in small amounts, during the formation of ATP, in the electron transport chain (ETC) by oxidative phosphorylation in mitochondria [19]. Many non-cellular processes like inflammatory reactions and ionizing radiations, and cellular processes like mitochondrial oxidative respiratory reactions, NADPH oxidases, and nitric oxide synthases (NOSs) contribute greatly to oxidative stress. These processes generate excess ROS resulting in imbalanced redox state and mitochondrial dysfunction. Hsp27 promotes a balanced redox state by reducing the ROS levels to avoid mitochondrial dysfunction, cell damage, and cell death [19].

\section{The convincing therapeutic potentials of Hsp27 in neurological diseases}

A study was performed to demonstrate Hsp27 neuroprotective effects by mutating serine residues to either alanine (Hsp27-A) or aspartate (Hsp27-D). In both in vivo and in vitro conditions, overexpression of Hsp27 wildtype and Hsp-D by phosphorylation provided neuroprotection by inhibiting the Ask-1 signaling pathway. In unphosphorylated form, it was insufficient to suppress the Ask-1 pathway or to provide protection [20]. In a study involving glial cell inclusion bodies, the response of Hsp27 to induced stress was studied. The bodies were 


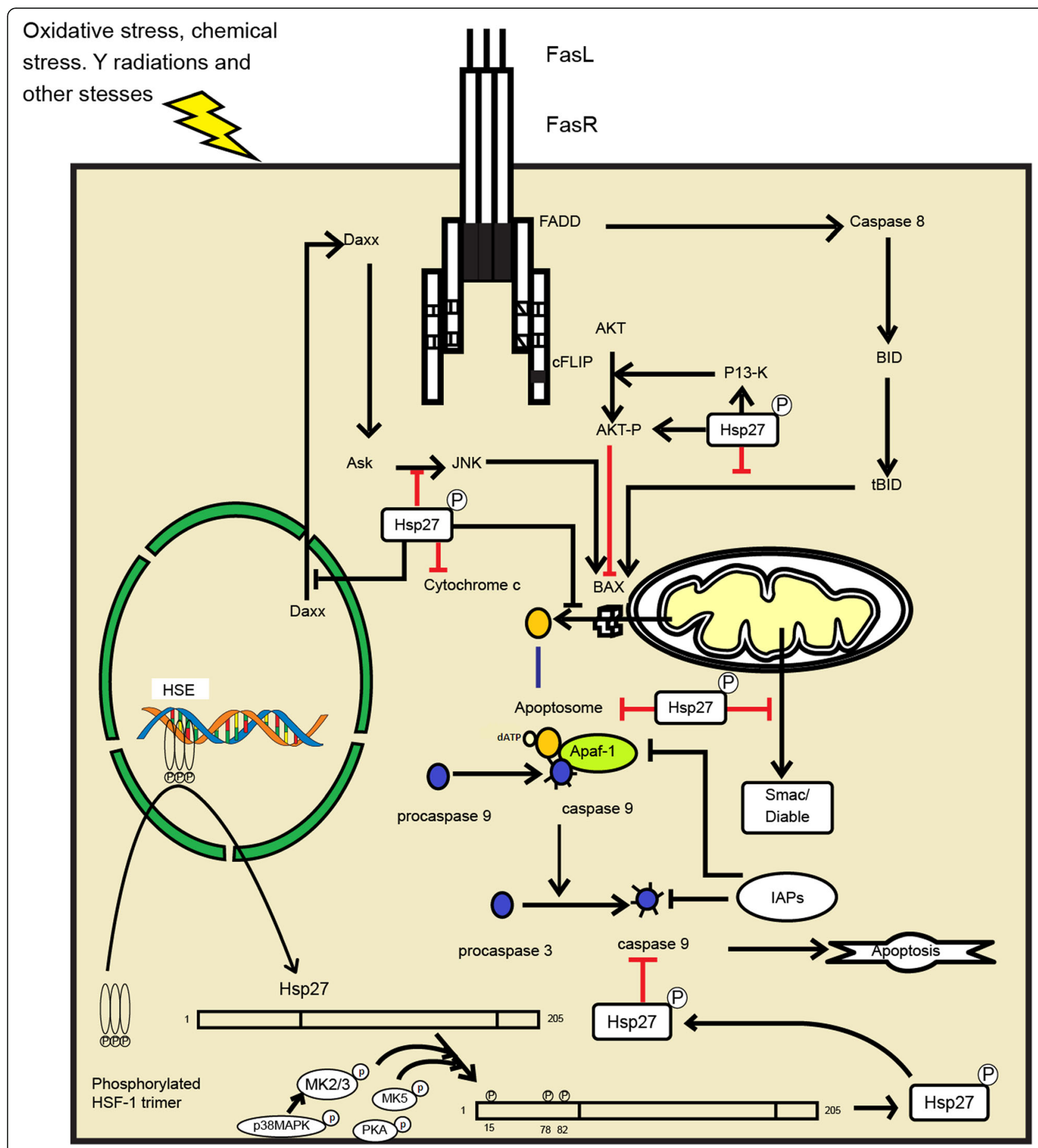

Fig. 1 Schematic representation showing the regulatory and inhibitory actions of Hsp27. Through induction of stress, the expression of hsp27 gene occurs when HSF-1 trimer binds to HSE. Hsp27 expression level gets varied when phosphorylated at specific serine residues such as Ser15, Ser78, and Ser82. Phosphorylated Hsp27 interferes with apoptotic signaling pathway by affecting its key components like cytochrome c,

apoptosome, Daxx, Smac, tBID, caspase 9, and ASK1-JNK through interaction with both mitochondria-dependent and mitochondria-independent pathways of apoptosis

transfected with OLN-3 cells carrying plasmids encoding Hsp27 expressed in three ways-wild-type, pseudophosphorylated form, and nonphosphorylatable form. The study revealed that Hsp27 regulated by phosphorylation protected the cytoskeleton and provided resistance from apoptotic stimuli upon stress conditions [21].

In another study, human neuroblastoma cell lines IMR-32 were treated with $\mathrm{Cu}^{2+}$ resulting in increased 
stress to the cells. This indirectly stimulated an increased level of endogenous Hsp27 and the overexpression of $\mathrm{Hsp} 27$, in turn, protecting the cells from $\mathrm{Cu}^{2+}$-induced cell death. Hsp27 hindered the upstream mechanism by inhibiting ROS production, decreasing the cascade events of $\mathrm{Cu}^{2+}$-induced inflammation and oxidative stress, and playing a protective role in maintaining $\mathrm{Cu}^{2+}$ homeostasis [22]. Studies have also shown that activated microglia-derived TNF $\alpha$ causes inflammation and degeneration processes [23]. When treated with Hsp27, which is an antioxidant, the cells were protected from TNF $\alpha$-mediated apoptosis by lowering the levels of reactive oxygen intermediate (ROI) formation by modulating glutathione content.

\section{Alzheimer's disease}

Alzheimer's disease (AD) is neuropathologically characterized by memory impairment, and it is the most common type of dementia which alters cognitive abilities. The characteristics observed in the brains of people with $\mathrm{AD}$ are amyloid plaques and neurofibrillary tangles [24]. The amyloid plaques are primarily composed of $A \beta 42$ (42 residues $\beta$-amyloid peptide) which is hydrophobic in nature. Increased amounts of insoluble $\beta$-amyloid peptides cause an imbalance between $A \beta$ production and clearance in the central nervous system [25]. The most important function of tau protein is providing stability and promoting assembly of microtubules. Neurofibrillary tangles in AD indicate neuronal dysfunction induced by hyperphosphorylation of tau which aggregates into insoluble paired helical filaments (PHFs) [26]. The altered structure of tau in $\mathrm{AD}$ is due to abnormal posttranslational modifications like hyperphosphorylation, acetylation, glycosylation, and truncation [27]. Tau hyperphosphorylation can also lead to several other events like $A \beta$-mediated toxicity, inflammation, an increase in oxidative stress, and covalent modifications of tau [28]. Increase in oxidative stress results in overproduction of ROS which in turn leads to a cascade of events ultimately ending in apoptosis [29].

Other than Hsp27, sHsps like Hsp20, Hsp22, and $\alpha$ crystallins also have similar functions as anti-apoptotic; chaperone activity and preventing cell death pathway through the effect of the sHsps varies widely. Table 1 gives a detailed account of the phosphorylation sites and the different modes of action against the aggregation of amyloid proteins. Though being small in size, Hsp27 has a wide range of therapeutic potential. Limited studies were focused on its therapeutic abilities on AD, but there have been reports that have shown a restoration in the amyloid plaque and tangle formation [31-34].

Research has shown that the inhibition of tau hyperphosphorylation restores neuronal dysfunction and modifies disease progression in $\mathrm{AD}$ which could be a therapeutic target for treatment. A study [35] in human neuroblastoma cell line SH-SY5Y was performed by inducing hyperphosphorylation of tau with okadaic acid. For effective delivery, Hsp27 protein was fused with HIV Tat protein (Tat-Hsp27) and introduced to the hyperphosphorylated tau aggregates where it resulted in a reduction of hyperphosphorylated tau levels conferring protection against apoptotic cell death. It was also demonstrated that without ubiquitination, degradation of hyperphosphorylated tau was carried out by Hsp27 [31]. Abisambra et al. confirmed that tau fibril formation can be prevented in in vitro condition by the addition of recombinant Hsp27. Similarly, he also verified that tau protein levels were reduced in in vivo conditions by overexpressing Hsp27 inhibiting the formation of tau fibrils [36]. A study by Chang et al. showed that synthetic indole derivatives upregulated Hsp27 expression which in turn reduced tau misfolding [32].

Several researches are also carried out to study the inhibition of $A \beta$ aggregation and to attenuate $A \beta$ toxicity with Hsp27 as the target protein. Hsp27 binds to $A \beta$ inhibiting its aggregation into mature fibrils [30]. A study was also performed to determine and compare the mechanism of interaction among three sHsps namely Hsp20 from $\mathrm{Ba}$ besia bovis, Hsp17.7 from carrot, and Hsp27 from humans and their capability in lowering toxicity of $A \beta$ aggregation. It was seen that Hsp27 interacts only at a later stage after forming Hsp27-A $\beta$ mixture thus inhibiting the formation of fibrils [37]. In a mouse model of $\mathrm{AD}$, overexpression of Hsp27 improved the learning abilities, increased the excitability of synaptic neurons, and decreased the $A \beta$ aggregates [33]. Additionally, it was also observed that the increase in Hsp27 expression after exercises prevented the aggregation of plaques and greatly improved brain function of elderly women [34].

The above studies exhibited that Hsp27 provides an almost untouched avenue for therapeutic intervention by exerting a beneficial effect in reducing oxidative stress as an antagonistic effect on apoptosis and its inhibitory actions through interaction with the amyloid formation and also in tau pathologies by providing neuronal protection.

\section{Parkinson's disease}

The deposition of $\alpha$-synuclein ( $\alpha$-syn) into fibrillar protein aggregates is the characteristics of many neurodegenerative diseases collectively called $\alpha$-synucleinopathies including Parkinson, dementia with Lewy bodies, and multiple system atrophy. $\alpha$-Synuclein is a neuronal protein found in presynaptic terminals which modulates the synaptic activities like neurotransmitter release and vesicular trafficking [38]. The $\alpha$-synuclein aggregation was sensitive to inhibition of autophagy and the proteasome which lead to an increase in proportions of $\alpha$-synuclein inclusion cells. Parkinson's disease (PD) is a neurodegenerative disorder 


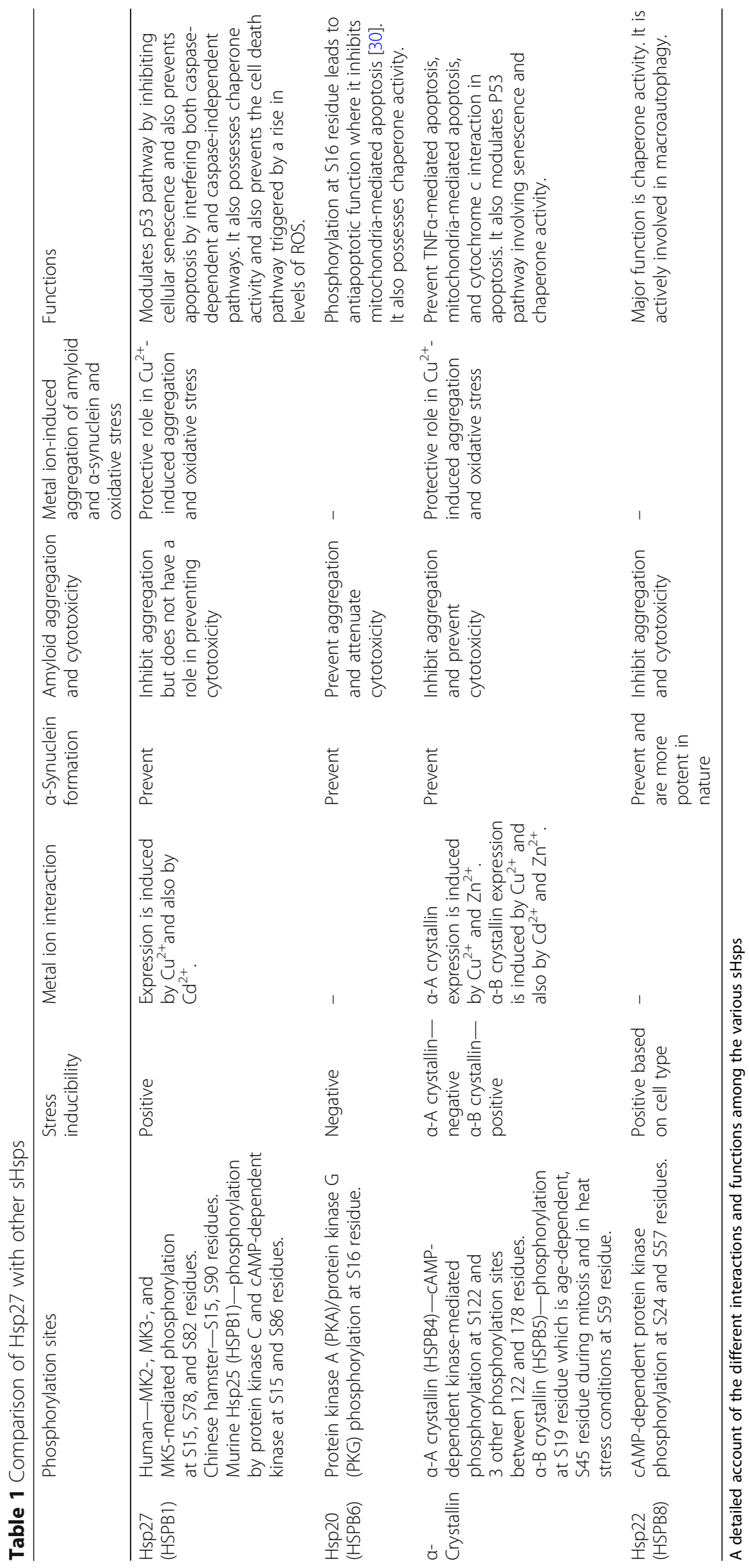


which is characterized by selective degeneration of dopaminergic neurons with Lewy bodies composed of $\alpha$ synuclein and sHsps [39].

Studies have revealed that sHsp upregulation in disease progression of $\mathrm{PD}$ prevents the degeneration of neurons [40]. Overexpression of $\alpha-B$ crystallin and Hsp27 [41] by using bicistronic expression plasmids prevented the intracellular aggregation of $\alpha$-syn. It was also suggested that the effectiveness of Hsp27 was dependent on the kinetics of $\alpha$-syn aggregation. Hsp27 was found to be less effective at a faster rate of aggregation [38]. A study showed that the expression of Hsp27/70 in SH-SY5Y cells induced by FLZ (a synthetic novel derivative of squamosamide from a Chinese herb) provided neuroprotective effects against $\mathrm{MPP}+$-induced cytotoxicity where MPP+ is a neurotoxin used in mimicking PD model [42].

A recent study revealed that Hsp27 binds to the $\alpha$-syn fibrils thus decreasing their hydrophobicity and cellular toxicity. It was also shown that Hsp27 was capable of inhibiting the elongation of $\alpha$-syn [43]. Additionally, Hsp27 also prevents the aggregation of monomeric $\alpha-$ syn fibrils along with $\alpha$-crystallins [38]. All these properties highlight the therapeutic potential of Hsp27 in preventing aggregation of $\alpha$-synuclein and the progression of $\alpha$-synucleinopathies.

\section{Therapeutic strategy of Hsp27 in other neurological diseases \\ Amyotrophic lateral sclerosis}

Hsp27 may also have a therapeutic role in amyotrophic lateral sclerosis (ALS). ALS is a rare neurodegenerative disorder characterized by progressive muscle weakness and atrophy due to the death of motor neurons in the spinal cord, cortex, and brainstem. In a study conducted, Hsp27 levels were increased and delivered to ND7 cells. When these cells were subjected to serum removal to induce apoptosis, overexpressed Hsp27 protected G93A or G93R SOD1 mutants from apoptotic cell death [44].

\section{Neuronal injury}

Neuronal injury after ischemia initiates a series of signaling cascades contributing to delayed neuronal death. In Hsp27 transgenic mice, overexpression of Hsp27 protected the cells against subsequent neuronal injury by inhibiting ASK1-dependent MKK4/JNK activation. This reflects that Hsp27 has a therapeutic potential during a stroke [45].

\section{Ataxia telangiectasia}

Ataxia telangiectasia is a neuromotor dysfunction neurodegenerative disorder of childhood caused by the disruption of gene ATM. In this disease, neurons lose their ability to divide and function. A study revealed that differential expression of Hsp27 in the frontal cortex can protect cortical neurons from degeneration, whereas in the cerebellum, proliferating glial cells were found to synthesize Hsp27 [46]. This again opens up the possibilities of using Hsp27 in treatment purposes.

\section{Charcot-Marie-Tooth disease}

Charcot-Marie-Tooth (CMT) neuropathies constitute a group of monogenic diseases that primarily affect the peripheral nervous system [47]. Mutations in Hsp27 can lead to adverse pathological neuromuscular disease as in distal hereditary motor neuropathy (dHMN) and also have been reported to cause CMT. These may be either due to toxic gain of function as a result of misfolding and aggregation or due to loss of function leading to decrease in the ability of cells to tolerate stress. Four mutant transgenic mouse models of dHMN were developed, and treatment with a selective HDAC6 inhibitor showed a reversal of the clinical phenotype of both S135F and P182L transgenic mice [48].

\section{Conclusion}

The therapeutic possibilities of Hsp27 are still not fully understood in most neurological diseases, but their role in these diseases cannot be ignored. Though not a lot of focus is placed on these small molecules, it is possible that these tiny molecules can shift the typical paradigm and open a new pathway to developing therapies for neurological diseases. Hsp27 has shown, in multiple diseases, that there is a chance to improve the condition of the cells affected and not just temporarily relieve the modifications. From the abovementioned studies, it is clear that Hsp27 may be a promising novel therapeutic target in treating neurological diseases.

\section{Abbreviations \\ AD: Alzheimer's disease; ALS: Amyotrophic lateral sclerosis; CMT: Charcot- Marie-Tooth; dHMN: Distal hereditary motor neuropathy; ETC: Electron transport chain; HSE: Heat shock element; HSF1: Heat shock transcription factor-1; HSF2: Heat shock transcription factor-2; Hsp: Heat shock protein; NOS: Nitric oxide synthase; PD: Parkinson's disease; PHF: Paired helical filament; ROI: Reactive oxygen intermediate; ROS: Reactive oxygen species; sHsp: Small heat shock protein; a-syn: a-Synuclein}

\section{Acknowledgements}

The authors would like to thank Bharathiar University, India, for providing the necessary infrastructure facilities required for completing the article.

\section{Authors' contributions}

AV and BV contributed to the concept development. AV and KS contributed to the manuscript writing and figure generation. BV contributed to the final approval. All authors have read and approved the manuscript.

\section{Funding}

Not applicable. This research did not receive any specific grant from funding agencies in the public, commercial, or not-for-profit sector.

\section{Availability of data and materials}

All data and materials are available in the manuscript and with the corresponding author.

Ethics approval and consent to participate Not applicable. 


\section{Consent for publication}

Not applicable.

\section{Competing interests}

The authors declare that they have no competing interests.

\section{Author details}

'Human Molecular Cytogenetics and Stem Cell Laboratory, Department of Human Genetics and Molecular Biology, Bharathiar University, Coimbatore, Tamil Nadu 641046, India. ${ }^{2}$ School of Biotechnology, Madurai Kamaraj University, Madurai, Tamil Nadu 625021, India.

Received: 19 June 2019 Accepted: 13 September 2019

Published online: 05 November 2019

\section{References}

1. Bakthisaran R, Tangirala R, Rao CM (2015) Small heat shock proteins: role in cellular functions and pathology. Biochim Biophys Acta BBA Proteins Proteomics 1854(4):291-319

2. Weeks SD, Muranova LK, Heirbaut M, Beelen S, Strelkov SV, Gusev NB (2018) Characterization of human small heat shock protein HSPB1 a-crystallin domain localized mutants associated with hereditary motor neuron diseases. Sci Rep 8(1):688

3. Feder ME, Hofmann GE (1999) Heat-shock proteins, molecular chaperones, and the stress response: evolutionary and ecological physiology. Annu Rev Physiol 61(1):243-282

4. Gomez-Pastor R, Burchfiel ET, Thiele DJ (2018) Regulation of heat shock transcription factors and their roles in physiology and disease. Nat Rev Mo Cell Biol 19(1):4

5. Wilkerson DC, Skaggs HS, Sarge KD (2007) HSF2 binds to the Hsp90, Hsp27, and c-Fos promoters constitutively and modulates their expression. Cell Stress Chaperones 12(3):283-290

6. Shiryaev A, Dumitriu G, Moens U (2011) Distinct roles of MK2 and MK5 in CAMP/PKA-and stress/p38 MAPK-induced heat shock protein 27 phosphorylation. J Mol Signal 6(1):4

7. Arrigo A-P (2017) Mammalian HspB1 (Hsp27) is a molecular sensor linked to the physiology and environment of the cell. Cell Stress Chaperones 22(4): 517-529

8. Gurgis FMS, Ziaziaris W, Munoz L (2014) Mitogen-activated protein kinaseactivated protein kinase 2 in neuroinflammation, heat shock protein 27 phosphorylation, and cell cycle: role and targeting. Mol Pharmacol 85(2): 345-356

9. Guay J, Lambert H, Gingras-Breton G, Lavoie JN, Huot J, Landry J (1997) Regulation of actin filament dynamics by p38 map kinase-mediated phosphorylation of heat shock protein 27. J Cell Sci 110(3):357-368

10. Vidyasagar A, Wilson NA, Djamali A (2012) Heat shock protein 27 (HSP27): biomarker of disease and therapeutic target. Fibrogenesis Tissue Repair 5(1):7

11. Lavoie JN, Hickey E, Weber LA, Landry J (1993) Modulation of actin microfilament dynamics and fluid phase pinocytosis by phosphorylation of heat shock protein 27. J Biol Chem 268(32):24210-24214

12. Zhu Y, O'Neill S, Saklatvala J, Tassi L, Mendelsohn ME (1994) Phosphorylated HSP27 associates with the activation-dependent cytoskeleton in human platelets. Blood 84(11):3715-3723

13. Akbar MT, Lundberg AM, Liu K, Vidyadaran S, Wells KE, Dolatshad H et al (2003) The neuroprotective effects of heat shock protein 27 overexpression in transgenic animals against kainate-induced seizures and hippocampal cell death. J Biol Chem 278(22):19956-19965

14. Havasi A, Li Z, Wang Z, Martin JL, Botla V, Ruchalski K et al (2008) Hsp27 inhibits Bax activation and apoptosis via a phosphatidylinositol 3-kinasedependent mechanism. J Biol Chem 283(18):12305-12313

15. Leak RK, Zhang L, Stetler RA, Weng Z, Li P, Atkins GB et al (2013) HSP27 protects the blood-brain barrier against ischemia-induced loss of integrity. CNS Neurol Disord-Drug Targets Former Curr Drug Targets-CNS Neurol Disord 12(3):325-337

16. Ikwegbue PC, Masamba P, Oyinloye BE, Kappo AP (2017) Roles of heat shock proteins in apoptosis, oxidative stress, human inflammatory diseases, and cancer. Pharmaceuticals 11(1):2

17. Charette SJ, Landry J (2000) The interaction of HSP27 with Daxx identifies a potential regulatory role of HSP27 in Fas-induced apoptosis. Ann N Y Acad Sci 926(1):126-131
18. Tian X, Zhao L, Song X, Yan Y, Liu N, Li T et al (2016) HSP27 inhibits homocysteine-induced endothelial apoptosis by modulation of ROS production and mitochondrial caspase-dependent apoptotic pathway. Biomed Res Int 2016:4847874

19. McCord MC, Aizenman E (2014) The role of intracellular zinc release in aging, oxidative stress, and Alzheimer's disease. Front Aging Neurosci 6:77

20. Stetler RA, Gao Y, Zhang L, Weng Z, Zhang F, Hu X et al (2012) Phosphorylation of HSP27 by protein kinase D is essential for mediating neuroprotection against ischemic neuronal injury. J Neurosci 32(8):2667-2682

21. Bolhuis S, Richter-Landsberg C (2010) Effect of proteasome inhibition by MG132 on HSP27 oligomerization, phosphorylation, and aggresome formation in the OLN-93 oligodendroglia cell line. J Neurochem 114(4):960-971

22. Asthana A, Bollapalli M, Tangirala R, Bakthisaran R, Rao CM (2014) Hsp27 suppresses the Cu2+-induced amyloidogenicity, redox activity, and cytotoxicity of a-synuclein by metal ion stripping. Free Radic Biol Med 72: 176-190

23. Nakajima K, Kohsaka S (2001) Microglia: activation and their significance in the central nervous system. J Biochem (Tokyo) 130(2):169-175

24. Takeda S, Sato N, Morishita R (2014) Systemic inflammation, blood-brain barrier vulnerability and cognitive/non-cognitive symptoms in Alzheimer disease: relevance to pathogenesis and therapy. Front Aging Neurosci 6:171

25. Mawuenyega KG, Sigurdson W, Ovod V, Munsell L, Kasten T, Morris JC et al (2010) Decreased clearance of CNS $\beta$-amyloid in Alzheimer's disease. Science 330(6012):1774-1774

26. Šimić G, Babić Leko M, Wray S, Harrington C, Delalle I, Jovanov-Milošević N et al (2016) Tau protein hyperphosphorylation and aggregation in Alzheimer's disease and other tauopathies, and possible neuroprotective strategies. Biomolecules 6(1):6

27. Gendron TF, Petrucelli L (2009) The role of tau in neurodegeneration. Mol Neurodegener 4(1):13

28. Ballatore C, Lee VM-Y, Trojanowski JQ (2007) Tau-mediated neurodegeneration in Alzheimer's disease and related disorders. Nat Rev Neurosci 8(9):663

29. Ferreira CA, Ni D, Rosenkrans ZT, Cai W (2018) Scavenging of reactive oxygen and nitrogen species with nanomaterials. Nano Res 11(10):4955-4984

30. Wilhelmus MM, Boelens WC, Otte-Höller I, Kamps B, de Waal RM, Verbeek MM (2006) Small heat shock proteins inhibit amyloid- $\beta$ protein aggregation and cerebrovascular amyloid- $\beta$ protein toxicity. Brain Res 1089(1):67-78

31. Shimura H, Miura-Shimura Y, Kosik KS (2004) Binding of tau to heat shock protein 27 leads to decreased concentration of hyperphosphorylated tau and enhanced cell survival. J Biol Chem 279(17):17957-17962

32. Chang K, Lin C, Chen H, Huang H, Chen S, Lin T et al (2017) The potential of indole/indolylquinoline compounds in tau misfolding reduction by enhancement of HSPB 1. Cns Neurosci Ther 23(1):45-56

33. Tóth ME, Szegedi V, Varga E, Juhász G, Horváth J, Borbély E et al (2013) Overexpression of Hsp27 ameliorates symptoms of Alzheimer's disease in APP/PS1 mice. Cell Stress Chaperones 18(6):759-771

34. Kim J-H, Jung Y-S, Kim J-W, Ha M-S, Ha S-M, Kim D-Y (2018) Effects of aquatic and land-based exercises on amyloid beta, heat shock protein 27, and pulse wave velocity in elderly women. Exp Gerontol 108:62-68

35. Choi S, Oh JH, Kim H, Nam SH, Shin J, Park J-S (2015) Protective effect of tat PTD-Hsp27 fusion protein on tau hyperphosphorylation induced by okadaic acid in the human neuroblastoma cell line SH-SY5Y. Cell Mol Neurobiol 35(7):1049-1059

36. Fontaine SN, Sabbagh JJ, Baker J, Martinez-Licha CR, Darling A, Dickey CA (2015) Cellular factors modulating the mechanism of tau protein aggregation. Cell Mol Life Sci 72(10):1863-1879

37. Lee S, Carson K, Rice-Ficht A, Good T (2006) Small heat shock proteins differentially affect $A B$ aggregation and toxicity. Biochem Biophys Res Commun 347(2):527-533

38. Cox D, Selig E, Griffin MD, Carver JA, Ecroyd H (2016) Small heat-shock proteins prevent a-synuclein aggregation via transient interactions and their efficacy is affected by the rate of aggregation. J Biol Chem 291(43):22618-22629

39. Kujawska M, Jodynis-Liebert J (2018) Polyphenols in Parkinson's disease: a systematic review of in vivo studies. Nutrients 10(5):642

40. Maiti P, Manna J, Veleri S, Frautschy S (2014) Molecular chaperone dysfunction in neurodegenerative diseases and effects of curcumin. Biomed Res Int 2014:495091

41. Cox D, Ecroyd H (2017) The small heat shock proteins aB-crystallin (HSPB5) and $\mathrm{Hsp}_{27}$ (HSPB1) inhibit the intracellular aggregation of a-synuclein. Cell Stress Chaperones 22(4):589-600 
42. Kong X, Zhang D, Qian C, Liu G, Bao X (2011) FLZ, a novel HSP27 and HSP70 inducer, protects SH-SY5Y cells from apoptosis caused by MPP+. Brain Res 1383:99-107

43. Cox D, Whiten DR, Brown JW, Horrocks MH, San Gil R, Dobson CM et al (2018) The small heat shock protein Hsp27 binds a-synuclein fibrils, preventing elongation and cytotoxicity. J Biol Chem 293(12):4486-4497

44. Patel YJ, Smith MDP, de Belleroche J, Latchman DS (2005) Hsp27 and Hsp70 administered in combination have a potent protective effect against FALSassociated SOD1-mutant-induced cell death in mammalian neuronal cells. Mol Brain Res 134(2):256-274

45. Stetler RA, Cao G, Gao Y, Zhang F, Wang S, Weng Z et al (2008) Hsp27 protects against ischemic brain injury via attenuation of a novel stressresponse cascade upstream of mitochondrial cell death signaling. J Neurosci 28(49):13038-13055

46. Chen W, Kuizon S, Chiou BL, Bolton DC, Pullarkat RK, Junaid MA (2009) Differential expression of small heat shock protein 27 (Hsp27) in ataxia telangiectasia brains. Neurochem Res 34(9):1658-1667

47. Venugopal A, Chandran M, Eruppakotte N, Kizhakkillach S, Breezevilla SC, Vellingiri B (2018) Monogenic diseases in India. Mutat Res Mutat Res 776:2331

48. Rossor AM, Morrow JM, Polke JM, Murphy SM, Houlden H, Laura M et al (2017) Pilot phenotype and natural history study of hereditary neuropathies caused by mutations in the HSPB1 gene. Neuromuscul Disord 27(1):50-56

\section{Publisher's Note}

Springer Nature remains neutral with regard to jurisdictional claims in published maps and institutional affiliations.

\section{Submit your manuscript to a SpringerOpen ${ }^{\circ}$ journal and benefit from:}

- Convenient online submission

- Rigorous peer review

- Open access: articles freely available online

- High visibility within the field

- Retaining the copyright to your article

Submit your next manuscript at $\boldsymbol{\nabla}$ springeropen.com 